\title{
Assessment of EGFR gene mutations in circulating free DNA in monitoring of response to EGFR tyrosine kinase inhibitors in patients with lung adenocarcinoma
}

\author{
Marcin Nicoś ${ }^{1,2}$, Kamila Wojas-Krawczyk ${ }^{1,3}$, Paweł Krawczyk ${ }^{1,4}$, Izabela Chmielewska ${ }^{1}$, \\ Magdalena Wojcik-Superczyńska ${ }^{1}$, Katarzyna Reszka ${ }^{3,5}$, Robert Kieszko ${ }^{1}$, Anna Góra-Florek ${ }^{6}$, \\ Małgorzata Dudek ${ }^{6}$, Daria Świniuch ${ }^{7}$, Wojciech Papiewski ${ }^{8}$, Paulina Całka ${ }^{9}$, Marzanna Ciesielka ${ }^{9}$, \\ Rodryg Ramlau ${ }^{7}$, Janusz Milanowski ${ }^{1}$
}

\author{
${ }^{1}$ Department of Pneumonology, Oncology and Allergology, Medical University \\ of Lublin, Lublin, Poland \\ ${ }^{2}$ Science for Life Laboratory, Department of Medical Biochemistry and Biophysics, \\ Karolinska Institutet, Stockholm, Sweden \\ ${ }^{3}$ Lublin Foundation for Cancer Patients "JestemnaTak", Poland \\ ${ }^{4}$ Polish Society of Clinical Oncology, Warsaw, Poland \\ ${ }^{5}$ GENIM LCC, Institute of Genetics and Immunology, Lublin, Poland \\ Independent Public Provincial Hospital named Jan Boży, Lublin, Poland \\ ${ }^{7}$ Department of Oncology, Poznan University of Medical Science, Poznan, Poland \\ ${ }^{8}$ Department of Pulmonology and Pulmonary Oncology of Mazovian Specialist \\ Hospital, Radom, Poland \\ ${ }^{9}$ Department of Forensic Medicine, Medical University of Lublin, Lublin, Poland
}

Submitted: 18 May 2018

Accepted: 14 August 2018

Arch Med Sci 2020; 16 (6): 1496-1500

DOI: https://doi.org/10.5114/aoms.2019.89217

Copyright (c) 2019 Termedia \& Banach

The presence of EGFR mutations in non-small cell lung cancer (NSCLC) patients is commonly evaluated in tissue material (surgery/biopsy) that may not be both representative of the overall genetic profile, especially for patients with heterogeneous cancer or distant metastases, and sufficient to perform a reliable genetic test (e.g. low tumor cell content). Therefore, a promising alternative in the analysis of EGFR profile is circulating free DNA (cf-DNA) that is released into peripheral blood from both normal and tumor cells that have undergone apoptosis or necrosis [1-5]. Molecular analysis of cf-DNA enables detection and monitoring of EGFR mutations, as well as detection of the acquired resistance for $1^{\text {st }}$ and $2^{\text {nd }}$ generation EGFR-tyrosine kinase inhibitors (TKIs) (erlotinib, gefitinib, afatinib and dacomitinib) caused by Thr790Met substitution that sensitizes NSCLC cells for $3^{\text {rd }}$ generation EGFR-TKIs (osimertinib, rociletinib) [1-3]. EGFR status in plasma or serum is highly concordant with the tumor cells and can be alternatively used in molecular analysis when material from tumor lesions cannot be obtained [1, 4, 5].

In the current study we analyzed the sensitivity of EGFR gene examination in liquid biopsy and utility of this material in monitoring changes in EGFR status during the EGFR-TKI therapy. The studied group included 23 Caucasian patients ( 8 male and 15 female, median age: $71 \pm 9$ years) with lung adenocarcinoma and known status of activating EGFR mutations (analyzed in formalin-fixed paraffin-embedded (FFPE) and cell blocks). The plasma samples were collected prior to the first EGFR-TKI administration and in 10 patients were re-obtained every 2 months until disease progression. Detailed characteristic of the studied group is presented in Table I and on Figure 1.

\author{
Corresponding author: \\ Marcin Nicoś \\ Department \\ of Pneumonology, \\ Oncology and Allergology \\ Medical University of Lublin \\ 8 Jaczewskiego St \\ 20-954 Lublin, Poland \\ E-mail: \\ marcin_nicos@interia.pl
}


Table I. Characteristics of the studied group

\begin{tabular}{|c|c|}
\hline Parameter & Value \\
\hline \multicolumn{2}{|l|}{ Gender: } \\
\hline Male, $n(\%)$ & $8(35)$ \\
\hline Female, $n(\%)$ & $15(65)$ \\
\hline \multicolumn{2}{|l|}{ Age: } \\
\hline Age median \pm SD (years) & $61 \pm 11$ \\
\hline$\geq 65, n(\%)$ & $17(78)$ \\
\hline$<65, n(\%)$ & $5(22)$ \\
\hline \multicolumn{2}{|l|}{ Smoking status: } \\
\hline Former smokers, $n(\%)$ & $13(56)$ \\
\hline Non-smokers, $n$ (\%) & $10(44)$ \\
\hline \multicolumn{2}{|l|}{ TNM: } \\
\hline II, $n(\%)$ & $1(4)$ \\
\hline IIIA, $n(\%)$ & $2(8)$ \\
\hline IIIB, $n(\%)$ & $1(4)$ \\
\hline IIIC, $n(\%)$ & $1(4)$ \\
\hline $\mathrm{IV}, n(\%)$ & $1(4)$ \\
\hline IVA, $n(\%)$ & $14(63)$ \\
\hline IVB, $n(\%)$ & $3(13)$ \\
\hline \multicolumn{2}{|l|}{ EGFR mutations: } \\
\hline Deletions in exon $19, n(\%)$ & $11(48)$ \\
\hline Substitution Leu858Arg in exon $21, n(\%)$ & $12(52)$ \\
\hline
\end{tabular}

The sensitivity of the ctEGFR Mutation Analysis Kit (Entrogen, USA) was $81.2 \%$ (9/11) for deletions in exon 19 and 83.33\% (10/12) for substitution Leu858Arg in exon 21. Moreover, we did not observe false positive results in control materials. The concordance between plasma and tissue samples reached $82.61 \%$ (19/23), which was lower than the concordance demonstrated by Xiong et al. (93.3\%), Reck et al. (89\%), Douillard et al. (94.3\%) and Mok et al. (88\%) [1, 2, 4, 5]. On the other hand, Yao et al. observed lower concordance $(78.21 \%)$ between plasma and tissue samples in next generation sequencing (NGS) analysis [6]. However, nowadays digital droplet PCR (dd-PCR), BEAMing PCR and NGS techniques provide the highest sensitivity for mutation analysis in plasma samples and they are the most recommended $[2,6,7]$.

In our study most of the plasma samples showed a low copy number (LCN) content of mutated DNA (mean: 7.44\%; range: 0.02-23.8\%). The content of mutated DNA in plasma samples was statistically lower than the content of mutated DNA in tissue material (Wilcoxon test: $p<$
0.000035). We observed a significant positive correlation between concentration of mutated DNA in plasma samples and NSCLC stage (Spearman test: $R=+0.53 ; p=0.01$ ). Patients with metastatic NSCLC had significantly higher content of mutated cf-DNA than patients with earlier stages of NSCLC (Mann-Whitney U-test: $p=0.02$ ). Zhu et al. suggested the discrepancies between tissue and plasma samples were due to LCN of mutant DNA in plasma [8]. Wei et al. demonstrated that decrease of mutated cf-DNA quantity was statistically significant at the beginning of the treatment, with the exception of patients who carried de novo Thr790Met mutation [9]. Zhu et al. noted decreased concentration of EGFR mutated cf-DNA in plasma during the therapy that significantly correlated with reduction of the tumor burden [10]. Mok et al. reported that continuous presence of EGFR mutations in cf-DNA was a negative predictive factor for overall survival (18.2 months vs. 31.9 months) and correlated with rapid tumor progression [1]. In our study, concentration of mutated cf-DNA dramatic decreased in the first 2 months of treatment and stabilized at a low level. Moreover it did not increase at the moment of clinical progression. Zhou et al. observed that content of mutated cf-DNA was the lowest at the moment of the best response to EGFR-TKIs and increased to the highest level during the progression of disease in some patients, while in the other group of patients the content of mutated cf-DNA did not increase and remained at a stable, low level [3]. Lee et al. showed that NSCLC patients who reached an undetectable level of mutated cf-DNA within the first 2 months of EGFR-TKI treatment had a significantly longer progression free survival (PFS) than patients without reduction of content of mutated cf-DNA (10.1 months vs. 6.3 months) [11]. Similarly, Xiong et al. observed that patients who had a negative result of EGFR analysis in plasma within one month of EGFR-TKI therapy had longer PFS than patients with a positive result of cf-DNA molecular examination (11 months vs. 6 months) [2]. Zhu et al. observed that higher pre-treatment content of mutated cf-DNA shortens PFS during EGFR-TKIs therapy, but reduction of its content to an undetectable level was associated with prolongation of PFS [10].

Evaluation of content of cf-DNA with Thr790Met substitution should be performed at the same time as molecular evaluation of tissue biopsy because de novo pre-existing tumor cells with Thr790Met mutation may worsen the prognosis in patients treated with EGFR-TKIs [6]. In our study, the pre-treatment concentration of cf-DNA with Thr790Met substitution was undetectable or at a very low level. Presence of this cf-DNA appeared during the therapy, without increase at the 
M. Nicoś, K. Wojas-Krawczyk, P. Krawczyk, I. Chmielewska, M. Wojcik-Superczyńska, K. Reszka, R. Kieszko, A. Góra-Florek, M. Dudek, D. Świniuch, W. Papiewski, P. Całka, M. Ciesielka, R. Ramlau, J. Milanowski

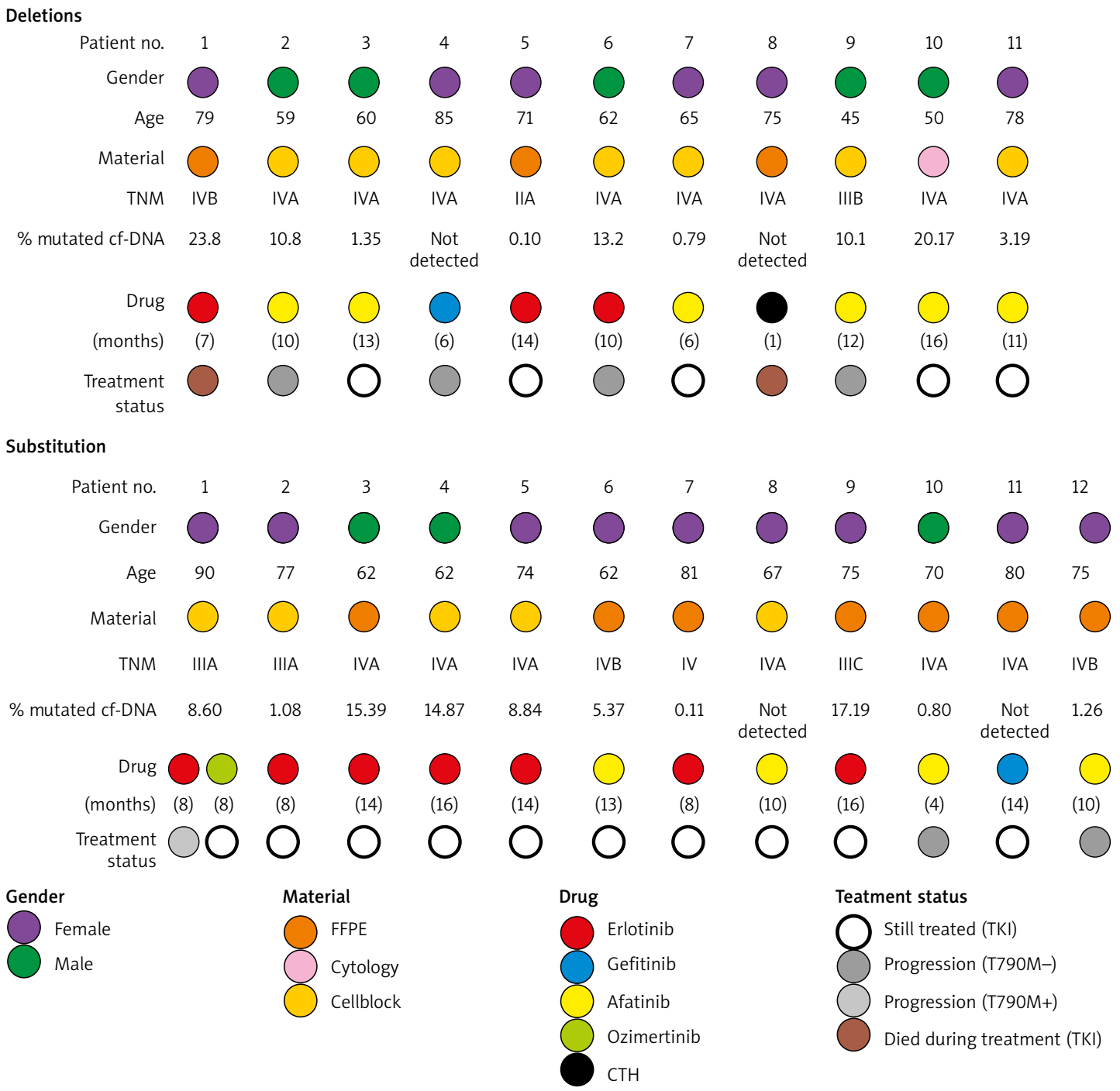

Figure 1. Clinical characteristics of the studied group

moment of progression. Presence of a low copy number of cf-DNA with a Thr790Met substitution did not affect the outcome of patients treated with $1^{\text {st }}$ and $2^{\text {nd }}$ generation EGFR-TKIs, with the exception of one female patient in whom a steady increase in the mutant DNA content coincided with the clinical progression of the disease. Finally, the patient responded to osimertinib with stabilization of mutant cf-DNA content (Figure 2). Zhu et al. mentioned that the overall incidence of de novo Thr790Met mutation in tumor tissues was $79.9 \%$, with higher frequency in larger tumors [9].

Xiong et al. observed dynamic changes in content of cf-DNA with different EGFR mutations in patients treated with $1^{\text {st }}$ and $2^{\text {nd }}$ generation EGFRTKIs. Among 24 patients who progressed during observation, 15 developed Thr790Met mutation, which was coexistent with an activating mutation, 7 patients lost EGFR gene mutations, 1 patient had only a Thr790Met substitution and 1 patient had only a primary deletion in exon 19.
Additionally, in 3 patients presence of Thr790Met substitution was observed without clinical progression [2]. Riediger et al. observed an increase in content of cf-DNA with Thr790Met mutation 2 months prior to clinical progression on EGFR-TKI therapy [12]. In the Zheng et al. study, high content of cf-DNA with Thr790Met mutation was observed 3 months before clinical progression on $1^{\text {st }}$ or $2^{\text {nd }}$ generation EGFR-TKIs and its concentration increased during 4-6 months prior to progression. Moreover, the content of cf-DNA with primary EGFR activating mutations also increased during clinical progression. Additionally, the presence of Thr790Met mutation in plasma was an independent prognostic factor for worse overall survival [13]. Thress et al. and Baijal et al. reported that molecular progression on EGFR- TKI treatment determined by Thr790Met detection in cf-DNA was determined 6 weeks earlier than clinical and radiological progression. Earlier administration of osimertinib according to 
A

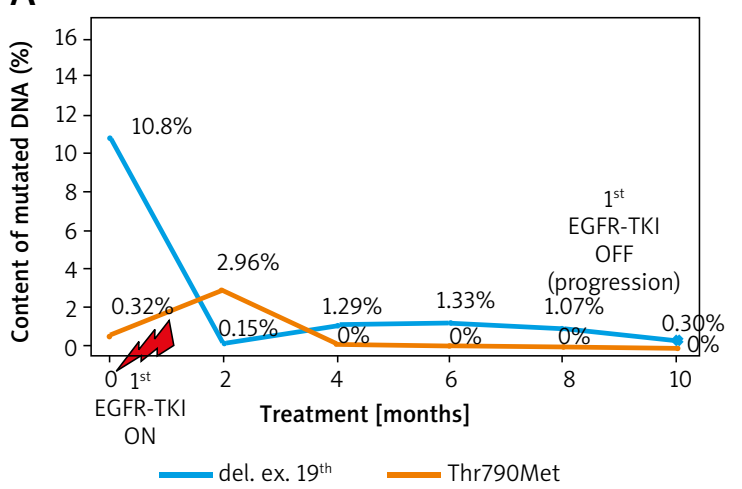

C

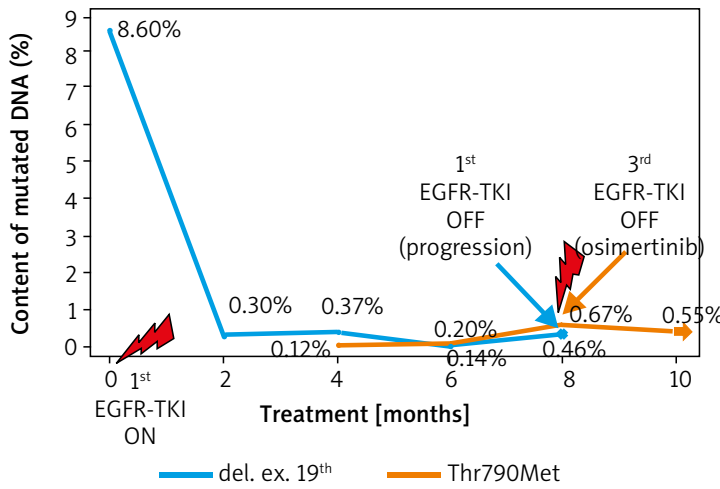

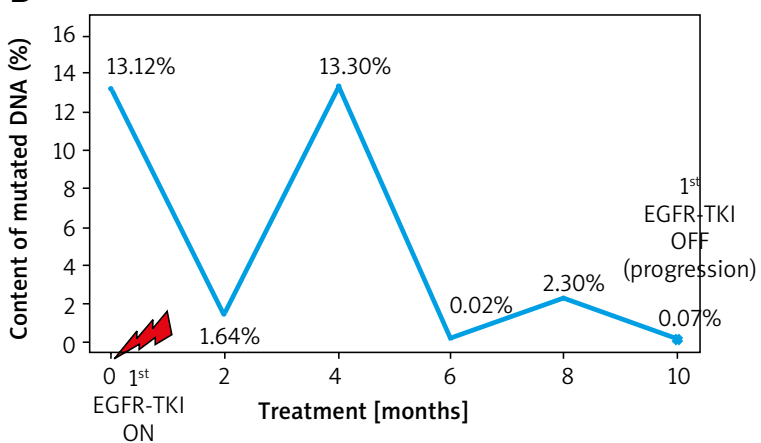

ON

del. ex. $19^{\text {th }}=$ Thr790Met (not detected)

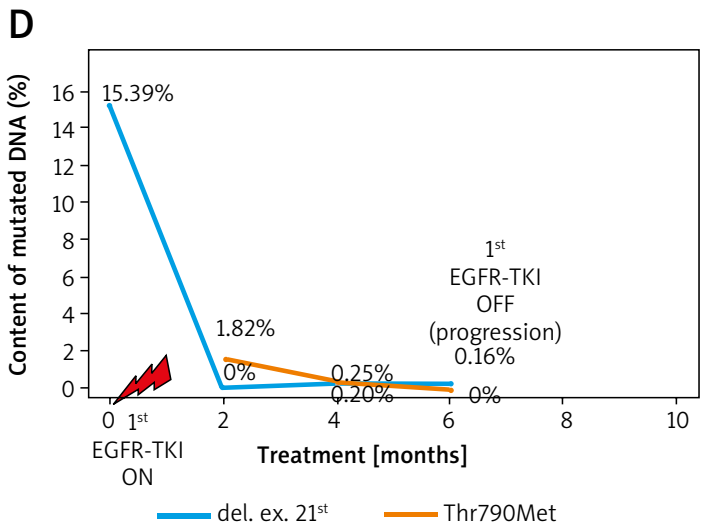

Figure 2. Changes in concentration of mutant DNA in plasma samples during EGFR-TKI treatment in four selected cases (A-D). Rapid decrease of mutated cf-DNA content within first 2 months of EGFR-TKI therapy and stabilization of mutated cf-DNA content at low level within next months of therapy. One patient showed an increase in concentration of mutated cf-DNA in the $4^{\text {th }}$ month of treatment (B) that could be caused by discontinuation and subsequent dose reduction of EGFR-TKI due to toxicity of therapy. When the recommended dose was administered, concentration of mutated cf-DNA reached a low level. The Thr790Met substitution was detectable from the beginning of the therapy or from the second month of this therapy, in the majority of patients. In 1 patient, cf-DNA with Thr790Met substitution was indicated at a detectable level after 4 months of treatment (C). Concentration of this cf-DNA steadily grew, reaching the highest level at the moment of clinical progression of the disease. At that moment, the content of cf-DNA with Thr790Met substitution was higher than the content of cf-DNA with exon 19 deletion. Therefore, the patient received osimertinib, which caused partial remission and stabilization of the disease

molecular findings of progression prolonged PFS (10.9 months vs. 5.5 months) and the overall response rate (70\% vs. $30 \%)$ to $3^{\text {rd }}$ generation EGFRTKIs in comparison to patients treated with osimertinib after confirmation of progression in radiologic examination $[14,15]$.

The study was approved by the Ethics Committee of the Medical University of Lublin, Poland (No. KE-0254/131/2011).

\section{Acknowledgments}

This work was supported by a START 2019 scholarship from the Foundation for Polish Science (FNP) to M.N.

\section{Conflict of interest}

The authors declare no conflict of interest.

\section{References}

1. Mok T, Wu YL, Lee JS, et al. Detection and dynamic changes of EGFR mutations from circulating tumor DNA as a predictor of survival outcomes in NSCLC patients treated with first-line intercalated erlotinib and chemotherapy. Clin Cancer Res 2015; 21: 3196-203.

2. Xiong L, Cui S, Ding J, et al. Dynamics of EGFR mutations in plasma recapitulates the clinical response to EGFR-TKIs in NSCLC patients. Oncotarget 2017; 8: 63846-56.

3. Zhou Q, Yang JJ, Chen ZH, et al. Serial cfDNA assessment of response and resistance to EGFR-TKI for patients with EGFR-L858R mutant lung cancer from a prospective clinical trial. J Hematol Oncol 2016; 9: 86.

4. Douillard JY, Ostoros G, Cobo M, et al. Gefitinib treatment in EGFR mutated caucasian NSCLC: circulating-free tumor DNA as a surrogate for determination of EGFR status. J Thorac Oncol 2014; 9: 1345-53.

5. Reck M, Hagiwara K, Han B, et al. ctDNA determination of EGFR mutation status in European and Japanese patients with advanced NSCLC: the ASSESS study. I Thorac Oncol 2016; 11: 1682-9. 
M. Nicoś, K. Wojas-Krawczyk, P. Krawczyk, I. Chmielewska, M. Wojcik-Superczyńska, K. Reszka, R. Kieszko, A. Góra-Florek, M. Dudek, D. Świniuch, W. Papiewski, P. Całka, M. Ciesielka, R. Ramlau, J. Milanowski

6. Yao Y, Liu J, Li L, et al. Detection of circulating tumor DNA in patients with advanced non-small cell lung cancer. Oncotarget 2017; 8: 2130-40.

7. Gu J, Zang W, Liu B, et al. Evaluation of digital PCR for detecting low-level EGFR mutations in advanced lung adenocarcinoma patients: a cross-platform comparison study. Oncotarget 2017; 8: 67810-20.

8. Zhu YJ, Zhang HB, Liu YH, et al. Estimation of cell-free circulating EGFR mutation concentration predicts outcomes in NSCLC patients treated with EGFR-TKIs. Oncotarget 2017; 8: 13195-205.

9. Wei Z, Wang W, Shu Z, Zhou X, Zhang Y. Correlation between circulating tumor DNA levels and response to tyrosine kinase inhibitors (TKI) treatment in non-small cell lung cancer. Med Sci Monit 2017; 23: 3627-34.

10. Zhu YJ, Zhang HB, Liu YH, et al. Association of mutant EGFR L858R and exon 19 concentration in circulating cell-free DNA using droplet digital PCR with response to EGFR-TKIs in NSCLC. Oncol Lett 2017; 14: 2573-9.

11. Lee JY, Qing X, Xiumin W, et al. Longitudinal monitoring of EGFR mutations in plasma predicts outcomes of NSCLC patients treated with EGFR TKIs: Korean Lung Cancer Consortium (KLCC-12-02). Oncotarget 2016; 7: 6984-93.

12. Riediger AL, Dietz S, Schirmer U, et al. Mutation analysis of circulating plasma DNA to determine response to EGFR tyrosine kinase inhibitor therapy of lung adenocarcinoma patients. Sci Rep 2016; 6: 33505.

13. Zheng D, Ye X, Zhang MZ, et al. Plasma EGFR T790M ctDNA status is associated with clinical outcome in advanced NSCLC patients with acquired EGFR-TKI resistance. Sci Rep 2016; 6: 20913.

14. Thress KS, Markovets A, Barrett JC, et al. Osimertinib, an EGFR T790M-selective tyrosine kinase inhibitor (TKI), provides high and durable response rates in patients (pts) with T790M positive advanced NSCLC whose disease has progressed following EGFR-TKI therapy. We investigated whether changes in the levels of plasma EGFRmutations post-osimertinib treatment are associated with clinical outcome. J Clin Oncol 2017; suppl 35: abstr. 9018.

15. Baijal S. Clinical utility of liquid biopsy in managing NSCLC patients. Performance and impact on clinical decisions. WCLC 2017. Abstract: SS 01.08. 